\title{
Stratégies de Revalorisation du Travail des Déchets Chez Les Pré-Collecteurs D’ordures Ménagères À Lomé
}

\author{
Akouété Galé Ekoué, Doctorant \\ Komitsè Edem Anika, Docteur \\ Komi Kossi-Titrikou, Professeur Titulaire \\ Unité de Recherche en Anthropologie Appliquée et Fondamentale \\ (URAAF) Université de Lomé, Togo
}

Doi:10.19044/esj.2019.v15n31p223 URL:http://dx.doi.org/10.19044/esj.2019.v15n31p223

\section{Résumé}

Le ramassage des ordures en tant que travail des déchets, reste dans beaucoup de sociétés, une profession socialement dégradante du fait de la manipulation de la saleté. Il constitue de ce fait un facteur de stigmatisation pour ceux qui l'exercent. À Lomé, cette stigmatisation touche les précollecteurs d'ordures surtout ceux utilisant des charrettes à traction humaine. Cet article a pour objectif d'analyser les stratégies adoptées par les précollecteurs pour revaloriser leur travail. Pour y parvenir, l'étude des perceptions que ceux-ci ont de leur travail ainsi que l'image qu'ils ont d'euxmêmes a été nécessaire. La théorie de l'identité sociale a été utilisée comme cadre théorique de référence. La recherche a été menée au moyen d'une méthode d'enquête qualitative qui a consisté en cinq mois d'observation des pré-collecteurs pendant leurs tournées de ramassage dans le quartier Wété, en trois focus-groups et 65 entretiens individuels. Il en ressort que les précollecteurs perçoivent leur travail comme dévalorisant, difficile et dangereux. Cependant, ils ont d'eux-mêmes une image gratifiante car surmontant toutes sortes de danger pour maintenir la propreté des maisons et de la ville. Les stratégies de revalorisation qu'ils développent sont une réponse à la stigmatisation sociale dont ils font l'objet. Elles consistent entre autres en des tournures langagières visant à dépouiller le vocabulaire professionnel des termes désagréables liés aux déchets.

Mots-clés: Pré-collecteurs, Ordure, Stigmatisation, Stratégie de revalorisation, Lomé 


\title{
Strategies for Upgrading Wastes Work Among Household Garbage Pre-Collectors in Lomé
}

\author{
Akouete Gale Ekoue, Doctorant \\ Komitse Edem Anika, Docteur \\ Komi Kossi-Titrikou, Professeur Titulaire \\ Unité de Recherche en Anthropologie Appliquée et Fondamentale \\ (URAAF) Université de Lomé, Togo
}

\begin{abstract}
As a work of wastes, garbage collection remains in many societies, a socially degrading profession due to the handling of dirt. In Lomé, this stigmatization affects garbage pre-collectors, especially those using humandrawn carts. This paper aims to analyze the strategies adopted by the precollectors to revalue their work. To achieve this, it was necessary to study the perceptions they have of their work and the image they have of themselves. The social identity theory has been used as a theoretical frame of reference. The research was conducted using a qualitative survey method that consisted of five months observing pre-collectors during their collection rounds in the Wété neighborhood, three focus groups and 65 individual interviews. It turns out that pre-collectors perceive their work as belittling, difficult and harmful. However, they have a gratifying esteem of themselves due to their capacity to face all kinds of danger to tidy the houses and the city. The upgrading strategies they develop are a response to the social stigmatization they undergo. They consist, among other things, of language skills which strip the professional vocabulary of unpleasant terms associated with wastes.
\end{abstract}

Keywords: Pre-collectors, Garbage, Stigmatization, Upgrading Strategies, Lomé

\section{Introduction}

La pré-collecte d'ordures est une activité consistant à collecter les déchets auprès des ménages moyennant une somme fixée proportionnellement à la quantité et à les acheminer vers les dépotoirs intermédiaires de la ville. Elle s'est développée à Lomé à partir de la fin des années 1990 avec la déliquescence des services municipaux en charge de la gestion officielle des déchets (Tiankpa, 2016). En tant que travail des déchets, elle reste comme dans beaucoup de sociétés, une profession socialement dégradante du fait de 
la manipulation de la saleté qui y est associée (Corteel \& Le Lay, 2011; Lhuillier, 2011; Philippot, 1997). De ce fait, les personnes qui s'y adonnent font souvent l'objet d'une stigmatisation qui se traduit par des insultes, du mépris et de la compassion (Florin, 2015; Lhuillier, 2005). cette stigmatisation touche les pré-collecteurs d'ordures surtout ceux qui utilisent les charrettes à traction humaine (Ekoué, 2019). Elle se manifeste par, entre autres, les accusations de vol de matériaux recyclables dans les maisons, le pincement du nez au passage du pré-collecteur et sa charrette d'ordures, les moqueries, les injures dont voici quelques exemples : les saligauds, les soulards, les vauriens, les voleurs, les délinquants, les misérables, les crève-la-faim, etc. (Ekoué, op. cit.). La reconnaissance par les populations de l'importance du travail des précollecteurs n'empêche pas pour autant leur stigmatisation. Malgré cette situation, ils continuent de vaquer quotidiennement à leur occupation et ne se résignent pas à occuper le statut marginal que la société leur attribue. Devant ces constats, la question suivante devient légitime : comment les précollecteurs d'ordures réagissent-ils face à la stigmatisation sociale liée à leur travail ? Cette question soulève des interrogations subsidiaires que voici : comment perçoivent-ils leur travail ? Quelle image ont-ils d'eux-mêmes et quelles actions cela induit-il ?

Pour répondre à ces interrogations, le présent article se donne pour objectif d'identifier et d'analyser les stratégies de revalorisation du travail de ramassage des ordures chez les pré-collecteurs de Lomé. Il s'agira spécifiquement (i) de recueillir leurs conceptions du travail des déchets, (ii) d'analyser les perceptions qu'ils ont d'eux-mêmes et (iii) de ressortir les actions découlant de ces perceptions.

\section{Cadre théorique}

En vue de rendre compte des stratégies mises en œuvre par les précollecteurs d'ordures pour revaloriser leur travail, la théorie de l'identité sociale de Tajfel \& Turner (1979) a été utilisée comme cadre théorique de référence. Cette théorie repose sur les trois principes suivants: (i) Les individus tentent d'acquérir ou de maintenir une identité sociale positive ; (ii) Une identité sociale positive résulte de comparaison favorable entre l'endogroupe et des exogroupes pertinents ; (iii) Lorsque l'identité sociale est insatisfaisante, les personnes tentent soit de quitter leur groupe pour rejoindre un groupe plus valorisé, soit de rendre leur groupe actuel plus positivement différent. Dans ce dernier cas où l'identité sociale est négative, les efforts des individus pour rendre l'endogroupe plus positif se traduisent par une créativité sociale qui est une stratégie cognitive et collective dont l'objectif est de réinterpréter le monde afin que l'ensemble du groupe vive mieux sa situation (Bourguignon \& Herman, 2007). 
La théorie de l'identité sociale permet dans cet article de présenter implicitement les pré-collecteurs d'ordures comme un groupe auquel est associé une identité sociale négative en raison du travail des déchets qu'ils font et de la stigmatisation sociale que cela entraine à leur égard. Elle permet surtout d'analyser les réactions de ces travailleurs face à cette stigmatisation en lisant dans leurs auto-perceptions et dans les stratégies de revalorisation, une réinterprétation de leur profession ainsi qu'une créativité sociale visant à rendre leur groupe positivement différent à travers un renversement du stigmate du déchet qui lui est attaché.

\section{Méthodologie}

\section{Présentation de la zone d'étude}

L'étude a été menée dans la commune de Lomé, capitale du Togo, située à l'extrême Sud-Ouest du pays. Cette commune est subdivisée en 5 arrondissements et s'étend sur une superficie d'environ 9000 hectares (Biakouyé, 2014). Elle forme avec la préfecture d'Agoè-Nyivé au nord, la préfecture du golfe au nord-ouest et à l'est, la grande agglomération de Lomé (Figure $\mathrm{n}^{\circ} 1$ ). Comme toute la partie sud du Togo, elle jouit d'un climat de type subéquatorial avec deux saisons pluvieuses et deux saisons sèches.

La commune de Lomé compte une population qui s'élevait à 839566 habitants en 2010 (RGPH4) ${ }^{35}$. La quantité de déchets produite par Lomécommune et son agglomération est estimée à près de 800 tonnes par jour (Koledzi, 2011). Ces déchets sont acheminés par les pré-collecteurs principalement vers les dépotoirs intermédiaires de la ville. Vingt-cinq (25) structures ont été retenues par la mairie pour la pré-collecte dans la commune, mais on dénombre plus d'une centaine d'associations qui opèrent dans l'informel, sans compter les pré-collecteurs indépendants.

\footnotetext{
${ }^{35}$ Quatrième recensement général de la population et de l'habitat
} 


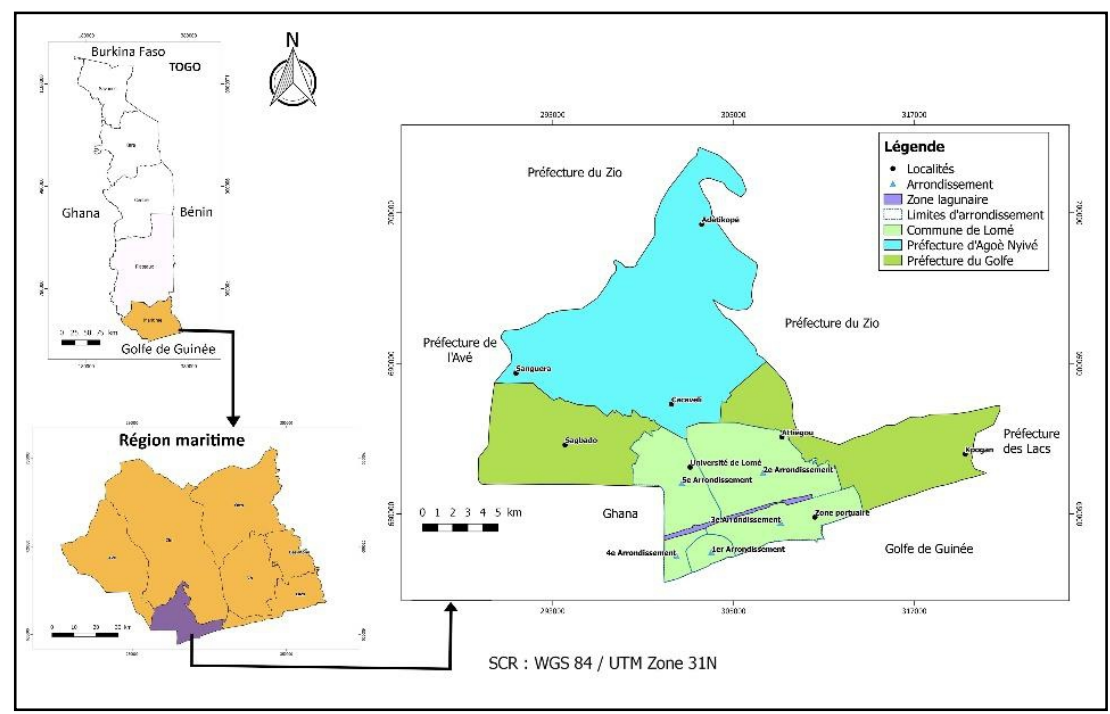

Figure $\mathbf{n}^{\circ} \mathbf{1}$ : Carte de situation de la ville de Lomé

Source : Direction nationale de la cartographie, 2017, adaptée par Ekoué, 2019

\section{Démarche}

Cette étude a pour public-cible les pré-collecteurs d'ordures à Lomé. Elle s'est particulièrement concentrée sur les pré-collecteurs utilisant les charrettes à traction humaine étant donné qu'ils subissent plus de stigmatisation que leurs collègues dotés d'engins motorisés comme l'a relevé Ekoué (op. cit.).

Pour atteindre les objectifs fixés dans le cadre de cette étude, une méthode d'enquête qualitative a été mise en place. Le choix de cette méthode s'explique par le fait qu'elle paraît la mieux adaptée pour cerner les stratégies de revalorisation du travail des déchets chez les pré-collecteurs de Lomé. De plus, elle permet d'étudier les conceptions que les pré-collecteurs ont de leur travail. Cette méthode repose sur des techniques classiques d'observation, de focusgroup, d'entretien individuel et de recueil de récits de vie.

Les observations ont été directes. Elles ont été réalisées dans le troisième arrondissement de la commune de Lomé précisément au quartier Wété, sur une durée de cinq (5) mois. Les faits observés sont essentiellement les interactions des pré-collecteurs avec les résidents du quartier et leurs comportements pendant les tournées de ramassage.

Trois focus-groups ont été réalisés avec les pré-collecteurs pendant leur pause de midi sur le dépotoir sauvage autorisé de Boka situé dans le quartier Akodessewa, à moins d'un kilomètre au nord du quartier Wété. Les discussions ont permis de recueillir les perceptions qu'ils ont de leur travail ainsi que l'image qu'ils ont d'eux-mêmes. En dehors de ces focus-group, des entretiens individuels ont été menés avec 26 pré-collecteurs non seulement sur 
le dépotoir de Boka mais aussi sur celui d'Amoutiévé situé dans le quartier du même nom. Au cours de ces entretiens, les récits de vie ont été recueillis ainsi que les difficultés relatives au travail de ramassage des ordures.

Des entretiens individuels ont été également menés auprès de 39 clients de pré-collecteurs. Les thèmes abordés lors de ces entretiens sont : les relations avec les ramasseurs d'ordures, leurs comportements pendant le service et les cas éventuels de conflits et leurs causes. Les entretiens ont été enregistrés sur autorisation de l'interlocuteur. En cas de refus de ce dernier, une prise de notes systématique a été effectuée.

L'ensemble des données recueillies a été retranscrit puis a fait l'objet d'une analyse thématique de contenu.

\section{Résultats}

\section{Pré-collecte d'ordures : un travail dévalorisant et risqué}

"Pour faire le travail de ramassage de déchets, tu dois avoir le cour, il faut être courageux! »; telle est la première phrase de Eugène, pré-collecteur de 31 ans, quand il a été interrogé sur le déroulement de l'activité de ramassage d'ordures.

En effet, le ramassage d'ordures est d'abord une activité dégradante et avilissante pour la catégorie socioprofessionnelle des pré-collecteurs. Ce caractère à la fois dégradant et avilissant vient du fait de gagner sa vie grâce aux ordures produites par les autres et surtout de manipuler quotidiennement cette saleté à laquelle les pré-collecteurs finissent par être identifiés. Aussi doivent-ils se confronter régulièrement à une stigmatisation qui se manifeste à travers les insultes, le manque de respect et les moqueries. De plus, ils doivent se surpasser et ignorer la honte qui est également associée à la manipulation des déchets dans l'espace public. En effet, le travail des déchets est considéré comme une activité honteuse surtout quand il s'effectue sous le regard des autres. Or, la pré-collecte des ordures est une activité qui se fait en plein jour, dans l'espace public sous le regard des clients et des passants. Un pré-collecteur rencontré devant le dépotoir d'Amoutiévé raconte :

«J'avais pris deux jeunes pour m'aider et à la fin de la journée, je leur trouvais quelque chose. Mais pendant les rondes, ils me disaient de ne pas passer ici, de ne pas passer là, parce qu'un membre de leur famille pourrait les voir, parce que leurs copines pourraient les voir (rire). Au bout de quelques semaines, ça m'a soulé et je les ai virés. Tu vois, ce travail, si tu ressens la honte, tu ne peux pas le faire » (23/12/2017). 
À la stigmatisation et la honte associée à la profession de ramasseur d'ordures, s'ajoute la difficulté du travail qui nécessite beaucoup d'efforts physiques pour tirer la charrette chargée de déchets (figure 2) sous le chaud soleil ou dans les rues inondées pendant la saison pluvieuse. Les conséquences pour ces pré-collecteurs sont essentiellement la fatigue, les courbatures et les pathologies musculo-squelettiques.

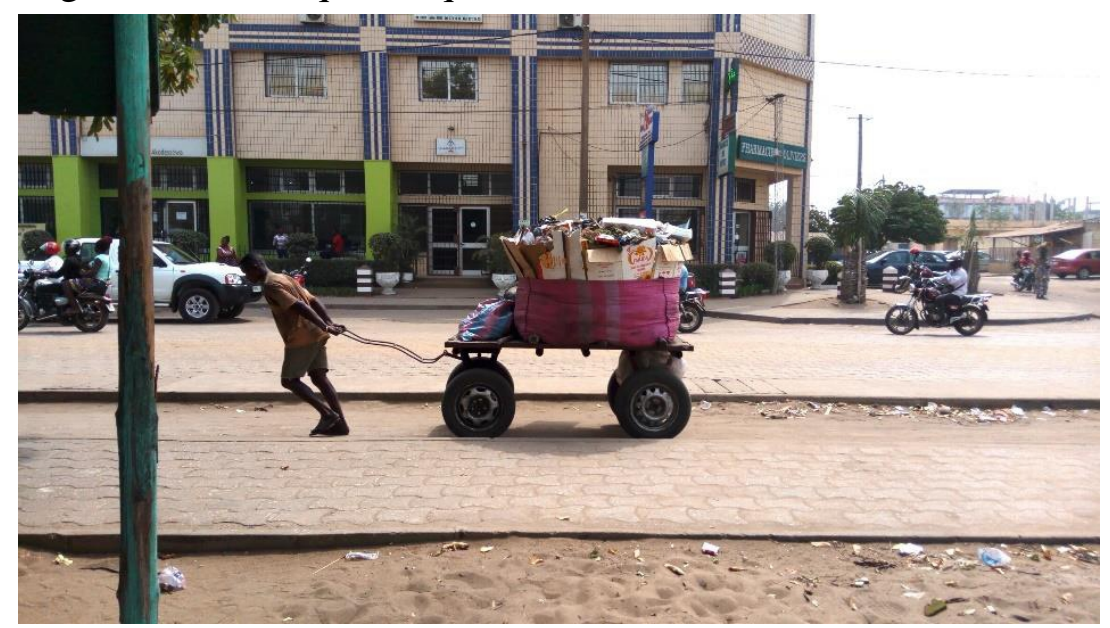

Figure 2 : Un pré-collecteur tirant sa charrette de déchets

Cliché : Ekoué, 2017

Le ramassage des ordures est ensuite pour les pré-collecteurs, une activité risquée voire dangereuse. Ce caractère dangereux est relatif d'une part, à la conception biomédicale associant le déchet aux maladies et d'autre part, à la pensée culturellement partagée selon laquelle les tas d'ordures sont le siège d'esprits malveillants. En effet, dans le ramassage des ordures, les risques d'accidents sont assez élevés. Que ce soit les blessures occasionnées par la charrette pendant sa traction ou celles causées par des objets tranchants contenus dans les ordures, les pré-collecteurs sont en permanence exposés aux accidents et aux maladies au cours de leur travail, dans la mesure où la plupart d'entre eux travaillent sans aucun matériel de protection. De plus, l'inhalation de la poussière et des mauvaises odeurs à longueur de journée n'arrange pas la situation. Les enquêtes ont révélé qu'il est fréquent de retrouver dans les ordures ramassées dans les maisons, des matières fécales emballées dans des sachets plastiques ou contenues dans des couches pour enfants. Parfois, ce sont des fœtus avortés, des gris-gris ou amulettes, des objets occultes qui y sont retrouvés. Ces amulettes et objets occultes sont considérés par les précollecteurs comme porteurs de charges spirituelles négatives pouvant nuire à celui qui les touche. Aussi, sur le plan spirituel, la proximité prolongée d'avec les déchets éloignerait-elle la chance et le bonheur de l'individu. C'est pour cela qu'il est important de se protéger mystiquement des ondes négatives émanant des esprits présents dans les immondices et également des effets des 
objets occultes qui y sont parfois trouvés. Chez certains pré-collecteurs, les tentatives de protection passent par le port de bracelets mystiquement préparés par des prêtres du culte des ancêtres, les promesses de dons ou de sacrifices à des divinités notamment Alafia kpeto deka ${ }^{36}$. Assou, un pré-collecteur exerçant depuis 13 ans, justifie ces stratégies de protection en ces termes : «Le déchet éloigne le bonheur et attire la malchance; et cette malchance nous touche, nous qui faisons le travail du déchet. C'est très dur de réussir quand tu fais ce travail » (26/12/2017). D'autres affirment ne pas avoir recours aux stratégies de protection évoquées ci-dessus. Ils préfèrent s'en remettre à la protection du Dieu des chrétiens auquel ils croient.

Les difficultés et la stigmatisation liées au ramassage des ordures font qu'il est considéré par les pré-collecteurs comme une activité de dernier choix. Ainsi, le ramassage des ordures ne devient une option envisageable qu'après avoir épuisé les recours possibles en vue d'une « meilleure profession ». Les récits de vie recueillis témoignent bien de cela. En voici un extrait :

«Avant de rentrer dans ce métier, je vivais dans la rue. En effet, je voulais que mon père m'envoie apprendre un métier, mais il a refusé en me disant de patienter pour que mes petitsfrères avancent dans leurs études pour atteindre le collège d'abord. Moi, je n'étais pas d'accord et à cause de ce différend, mon père a fini par me jeter hors de la maison. C'est ainsi que j'ai vécu dans la rue pendant 7 ans. Pour manger, je mendiais. Souvent, je me rendais dans un atelier de couture pour quémander quelques pièces pour manger. Un jour, un des apprentis me dit : toi aussi, au lieu de mendier tout le temps, va chercher un travail à faire et arrête de nous déranger ici. Cette phrase m'a tellement touché que j'ai commencé à réfléchir à quoi faire pour gagner de l'argent. Avec le conseil de quelques connaissances, j'ai commencé ce travail en 1999 » (Alex, 42 ans, 26/12/2017).

Si le ramassage des ordures est un travail de dernier recours, il est également un travail dans lequel il ne faut pas s'éterniser. Bien que ce travail leur garantisse des revenus, les pré-collecteurs sont unanimes pour attribuer à leur profession un caractère temporaire. Ainsi, un travail impliquant un contact direct avec les déchets comme le leur, ne devrait-il pas être exercé pendant très longtemps. S'ils ne précisent pas le nombre exact d'années à effectuer avant de se retirer, les pré-collecteurs considèrent leur travail comme un moyen pour gagner de l'argent, faire des économies et entreprendre autre

\footnotetext{
${ }^{36}$ Alafia kpeto deka est une des divinités les plus répandues chez les peuples du Sud Ghana, Togo et Bénin. Encore appelée Goro vodou (divinité de la cola), elle est réputée pour son pouvoir anti-sorcellerie, sa puissance thérapeutique en matière de fécondité, et sa capacité d'action dans les domaines de la réussite sociale et de la prospérité financière (Poirée, 2004).
} 
chose. Les différents projets d'avenir recensés grâce aux récits de vie ne prennent pas en compte le métier de pré-collecteur. Les activités envisagées pour le futur s'inscrivent essentiellement dans la logique d'entreprendre une activité commerciale.

\section{Pré-collecte d'ordures : un travail difficile fait par des "braves "}

En considérant la stigmatisation, les difficultés et les risques liés à l'exercice de la profession de ramassage d'ordures, les pré-collecteurs se perçoivent comme des gens courageux qui bravent le danger pour rendre service à la société. À travers leur métier, ils viennent en aide aux populations en les débarrassant de leurs restes et de ce fait, le caractère nécessaire de leur travail ne fait aucun doute. Ainsi, en réponse à la marginalisation dont ils sont l'objet, les pré-collecteurs se définissent non comme des miséreux mais plutôt comme des "sauveurs" de la population. Ils sauvent les citadins, non seulement des maladies que peuvent causer les déchets mais aussi, des entités maléfiques qui s'y refugient et ce, au péril de leur propre santé et bonheur. C'est ce que Togbé affirme avec une certaine fierté : «Par la pré-collecte des déchets, on ramasse les mauvais esprits dans la maison des gens. On est courageux, $n$ 'importe qui ne peut pas faire ça » (55 ans, pré-collecteur depuis 20 ans, dépotoir de Boka, 18/12/2017). Selon un autre,

«La saleté n'est pas une bonne chose, c'est pourquoi les gens veulent éloigner ça d'eux. Nous, on intervient pour sauver en quelque sorte les gens de leurs déchets. Des fois quand on ne passe pas et que les ordures s'accumulent chez les clients, ils sont inquiets. Dès qu'on apparaît on voit à quel point ils sont soulagés de nous voir. Donc je dirai que nous, on fait un travail très important car on vient débarrasser la population de la menace des déchets »(Chinois, 38 ans, dépotoir d'Amoutiévé, 27/12/2017).

Acceptant leur situation de pauvreté, les pré-collecteurs voient dans le travail des déchets une échappatoire pour ne pas tomber dans les travers de l'oisiveté, du vol et du banditisme. Aussi se considèrent-ils comme des gens qui s'efforcent de gagner honnêtement leur vie, des personnes avec des valeurs morales comme l'honnêteté, l'intégrité, la persévérance et le courage. Forts de ces auto-perceptions gratifiantes, ils développent des stratégies pour donner de la valeur et de l'importance à leur profession.

\section{Sortir de la marginalisation : quand les pré-collecteurs revalorisent leur travail}

Les stratégies mises en place par les pré-collecteurs à Lomé se fondent sur les perceptions essentiellement positives qu'ils ont d'eux-mêmes. Elles ont pour but de rappeler aux populations l'importance sociale et environnementale 
de leur profession et de sortir des catégories marginales dans lesquelles la société les confine. Ces stratégies sont de divers ordres.

D’abord on note chez les pré-collecteurs une utilisation de plus en plus courante de l'euphémisme dans le langage propre à leur travail ainsi qu'à une «positivation » de la profession. Comme cela a été déjà dit, ils se considèrent comme des "sauveurs", des bienfaiteurs de la société. Cette image qu'ils ont d'eux-mêmes, les amène à se vanter de faire un travail nécessaire pour la ville dans le sens où ils participent grandement à sa propreté. Pour eux, le ramassage des ordures n'est plus un sale boulot, mais plutôt un travail dont l'objectif est de rétablir la propreté des habitations et de maintenir la salubrité de la ville. C'est pourquoi, il est très fréquent de croiser dans les rues de Lomé, des précollecteurs qui proposent leurs services aux populations aux cris de : «axoea me ne ko ! axoea me ne ko! egbe ko to va, mia ks gbea ? ». Une traduction littérale de cette phrase faite en éwé37 donne ceci : "Que la maison soit propre! que la maison soit propre! le ramasseur de déchets est là, voulezvous évacuer vos ordures? ». Non seulement elle met en avant le rôle positif des pré-collecteurs, mais aussi elle démontre l'importance et la valeur que ceux-ci donnent à leur métier. Ces professionnels des déchets s'attribuent le statut d' " agents de propreté » au détriment de celui de « ramasseurs d'ordures ». C'est ce que semble défendre Agossa lorsqu'il affirme : "C'est grâce à nous que les maisons sont propres. Tu imagines la ville sans nous ? On dormirait tous dans les ordures. Même les camions de la voirie ne peuvent pas tout ramasser » (pré-collecteur, 38 ans, 04/01/2018).

Ensuite, les ramasseurs d'ordures dans leurs tentatives de revalorisation de leur métier mettent l'accent sur l'aspect économique de leur travail. Certes, le ramassage des ordures est considéré comme une activité de subsistance, mais il permet de faire face à la pauvreté et de s'assurer un revenu minimal au quotidien. Les enquêtes ont révélé qu'il permet aux pré-collecteurs de gagner en moyenne $5000 \mathrm{~F}$ CFA les jours où la demande est forte, ce qui n'est pas une somme dérisoire dans le contexte loméen où le niveau de vie est relativement bas comparé aux autres villes de la sous-région. Pour cela, ils se comparent sur le plan salarial au fonctionnaire moyen du secteur public. « Quand il y a du travail je fais jusqu'à 8000 F par jour. Je peux dire que je gagne comme un fonctionnaire voir plus ! C'est grâce à ce travail que j'aide mes petits frères à aller à l'école étant donné que je suis l'aîné » déclare un pré-collecteur (Dodzi, 26 ans, 16/12/2017) venu vider sa charrette au dépotoir de Boka.

Enfin, les pré-collecteurs, conscients de la position «stratégique » qu'ils occupent au sein de la société, usent de la technique du refus comme un moyen de se revaloriser aux yeux des populations. Cette technique consiste à

${ }^{37}$ L'éwé est la langue parlée à Lomé et dans toute la partie sud du Togo et même au-delà. 
refuser de ramasser les ordures chez certains clients ${ }^{38}$. C'est une stratégie qui est développée en réaction au manque de respect, aux accusations de vol, aux insultes, etc., dont ils font souvent l'objet dans l'exercice de leur profession mais aussi, aux ménages qui y mettent souvent des matières considérées comme impures à l'instar des matières fécales. Dans certains cas, il s'agit d'une mesure de protestation contre la fixation du prix de la prestation par les clients, ce qui est souvent en leur désavantage. En effet, les pré-collecteurs choisissent parfois de ne pas ramasser les ordures d'un client quand celui-ci tente d'imposer un prix beaucoup plus bas que celui qu'ils ont fixé. Dans ce cas de figure, deux situations se présentent : soit le pré-collecteur s'en va après une dispute ou non avec le client, soit des négociations s'engagent entre les deux acteurs. Dans ces négociations, les deux parties cherchent à trouver un accord sur le prix. Pendant que le client se plaint du manque d'argent en suppliant le pré-collecteur d'accepter la somme qu'il lui propose, le précollecteur utilise lui aussi des arguments similaires en y ajoutant la difficulté du travail pour amener le client à augmenter un peu son prix. Ces négociations ont habituellement lieu dans une ambiance détendue où les clients cherchent à amadouer les pré-collecteurs en faisant des blagues du genre : «mon mari, ne sois pas si dur avec moi, pardon»; «tu es mon gendre hein! Je vais te donner ma fille en mariage! » «On est ensemble depuis longtemps, fais-moi cette faveur », etc. Au fil du temps et des interactions, les négociations contribuent à établir le respect et une confiance mutuels entre les deux acteurs, créant un lien social durable, qui amène les pré-collecteurs à accorder occasionnellement des faveurs aux clients qu'ils jugent dignes. Ces faveurs consistent au ramassage d'ordures à crédit auprès de clients qui s'engagent verbalement à payer la prestation dans un délai arrêté de façon consensuelle. Ces délais peuvent varier du prochain passage du pré-collecteur à une semaine ou à un mois.

\section{Discussion}

Le caractère pénible du travail des déchets notamment le ramassage des ordures vient non seulement des risques encourus et des efforts physiques fournis, mais aussi de la stigmatisation sociale qui y est liée. En clair, les précollecteurs dans la réalisation de leur travail, doivent courir tout au long de la journée des risques professionnels de toutes sortes et supporter au même moment, le poids psychologique des pratiques de stigmatisation de la part des populations. Les résultats présentés dans cet article montrent l'effectivité de cette pénibilité du point de vue des pré-collecteurs d'ordures rencontrés à

\footnotetext{
${ }^{38}$ Sous le vocable « clients » sont regroupés les ménages, les restaurations de rue et autres lieux d'activités dont les déchets sont assimilés aux ordures ménagères. La catégorie « client » est généralement représentée par les femmes car elles sont en charge de la gestion domestique des déchets. C'est donc avec elles que les pré-collecteurs interagissent le plus souvent.
} 
Lomé. Ils reconnaissent le caractère dévalorisant et dégradant de leur travail, le ramassage des ordures étant négativement perçu car, le déchet «marque $d u$ sceau de l'abjection ceux qui le touchent, le manipulent » (Zonabend, 1999 : 90). Ce « sceau de l'abjection» dont ils sont marqués devient alors un stigmate important qui favorise des comportements de mépris à leur égard. Dans son étude sur les éboueurs de Sao Paulo, Soares (2011) analyse l'humiliation subie dans leurs interactions avec la population comme une source de souffrance au travail. Cette humiliation provient du dégoût que les éboueurs, de même que les égoutiers et les employés de morgue (Jeanjean, 2011b) suscitent chez la société environnante. Cette question de l'humiliation au travail amène à aborder la honte sociale qui est associée à la saleté et sa manipulation et donc au travail «dévalorisant » des déchets.

Comme cela a été relevé dans cette étude, manipuler le rebut se révèle être une activité honteuse surtout lorsqu'on considère la présence et le regard d'autrui. Ainsi, la honte a toujours accompagné la dévalorisation sociale du travail des déchets (Guitard, 2016). Lorsqu'on sort du domaine professionnel pour entrer dans le quotidien des ménages, l'évacuation des déchets sur le dépotoir est entachée par le même sentiment de honte (Bouju \& Ouattara, 2002 ; Guitard, 2015). C'est une des raisons pour lesquelles cette activité est confiée la plupart du temps aux femmes, chargées traditionnellement de la propreté de la concession et également aux enfants.

En plus du stigmate que constitue le déchet pour les pré-collecteurs, le ramassage d'ordures est une activité difficile et dangereuse compte tenu des risques d'accident et de contamination élevés. En effet, le métier de précollecteurs d'ordures, ici, tout comme celui des équipiers de collecte (Morlet, 2011), est caractérisé par une pénibilité dont la première manifestation est l'effort physique intense. Hormis les efforts physiques et les accidents, les déchets constituent une source de danger pour ceux qui les manipulent. Ceci s'explique par le fait qu'ils renferment des agents biologiques entre autres les bioaérosols, facteurs de problèmes pulmonaires, gastro-intestinaux et cutanés (Poulsen et al., 1995) et les germes pathogènes responsables de diverses maladies hydriques (Bagalwa et al., 2013). Les différents éléments présentés ici concordent donc avec les conceptions biomédicales que les pré-collecteurs loméens ont des déchets.

Parallèlement à ces conceptions inspirées du domaine biomédical, des perceptions socioculturelles faisant du déchet ou des tas d'ordures un refuge de forces surnaturelles négatives, traversent toute la société y compris les précollecteurs rencontrés. Ces perceptions viennent ainsi accentuer le danger que représente le déchet dans le sens où ce n'est plus seulement le corps qui est menacé mais l'esprit aussi. Elles ne sont pas présentes seulement au Togo mais répandues dans plusieurs sociétés africaines. À Kinshasa en République Démocratique du Congo, Lelo Nzuzi (2008) relève chez les populations, des 
croyances qui considèrent les décharges anarchiques comme des foyers d'esprits impurs. Dans la même veine, (Guitard, 2012, 2014) découvrent à Maroua et Garoua au nord du Cameroun, des perceptions qui attribuent au tas d'ordures la faculté d'abriter une « force morbide » et d'attirer des entités malveillantes.

Telles que présentées au niveau des résultats, les perceptions que les travailleurs des déchets, en l'occurrence les pré-collecteurs, ont d'eux-mêmes, sont marquées par une certaine positivité malgré le « mauvais » regard que la société porte sur cette profession. Non seulement ils insistent sur le caractère nécessaire de leur travail mais surtout, ils se perçoivent comme des hommes de courage et des sauveurs des populations. Ces perceptions rejoignent celles relevées par Jeanjean (2001) chez les égoutiers de Montpellier. En effet, l'auteur arrive à dégager à travers l'analyse des discours tenus par ces égoutiers, des auto-perceptions qui sont caractérisées par la force, le courage et l'héroïsme dont ils font preuve au cours de leur travail. C'est en suivant cette logique qu'ils mettent en place des stratégies pour « subvertir le "sale boulot" en bon boulot, pour transformer la honte en fierté » (Corteel \& Le Lay, 2011: 27) et par là, se « réapproprier une dignité » (Bercot, 2011 : 210). Rappelons que dans cet article, les stratégies utilisées par les pré-collecteurs de Lomé pour atténuer la dévalorisation sociale dont ils font l'objet ont été mises en lumière. Il s'agit essentiellement de l'utilisation de l'euphémisme et la «positivation » de la profession, la mise en avant du gain financier et le refus du ramassage d'ordures conduisant à des négociations. Plusieurs auteurs ont également abordé la question des stratégies de revalorisation notamment les manipulations langagières, les techniques de «positivation » de la profession et le recours aux valeurs morales dont les travailleurs des déchets font usage. Dans son analyse du « sale boulot », Lhuilier (2005) évoque chez les professionnels de l'ordure, la revendication d'un professionnalisme illustré par des compétences techniques doublées d'une rigueur éthique. La dimension morale soulevée ici, rejoint nos résultats dans le sens où les pré-collecteurs mettent en avant une série de valeurs morales, en réaction à la stigmatisation sociale et à la difficulté de leur travail. La même remarque est faite par Jeanjean (2011a) chez les agents de morgue et les égoutiers qui mobilisent des valeurs et une éthique pour faire face au dégoût exprimé par le public. À part le recours à l'éthique, les travailleurs des déchets opèrent une atténuation de leur langage afin de renverser le stigmate de l'ordure. C'est dans cette logique que Lhuillier (2005) souligne l'adoption par les professionnels de l'ordure, d'un vocabulaire euphémique visant à neutraliser «la charge affective associée à l'univers de l'ordure » (op cit: 81). L'auteur donne l'exemple des «entrepreneurs de boue » qui sont désormais appelés «industriels de la dépollution » et « du balayeur » que l'on désigne maintenant par le terme « technicien de surface ». Dans le même sens, Guitard (2016) observe chez les 
éboueurs au nord du Cameroun des postures et des contournements qui contribuent à les faire passer du statut de « ramasseurs d'ordures » à celui de « commandeurs de la propreté ». C'est ainsi que des termes considérés comme plus sains viennent remplacer ceux liés à la saleté. Tel est le cas de l'expression «mettre la propreté » qui est utilisée à la place de « enlever les ordures ».

\section{Conclusion}

En réponse à la stigmatisation sociale dont ils font l'objet, les précollecteurs d'ordures développent une diversité de stratégies pour revaloriser leur travail. Cet article a permis de passer en revue et d'analyser ces stratégies en étudiant au préalable, les perceptions que ces pré-collecteurs ont de leur travail et l'image qu'ils ont d'eux-mêmes. Ainsi, la pré-collecte d'ordures est aux yeux de ceux qui l'exercent, un travail dévalorisant, difficile et dangereux mais qui leur permet de gagner honnêtement leur vie. La dévalorisation se rapporte à la stigmatisation essentiellement due à la manipulation de la saleté et des matières déchues appartenant à d'autres. La difficulté tient de l'exigence physique que requiert l'activité et des nombreux accidents auxquels sont exposés les travailleurs. Le danger est surtout lié aux déchets dans ce sens qu'ils renferment des agents pathogènes responsables des diverses maladies (conception biomédicale) mais aussi, parce qu'ils constituent un refuge pour les esprits malveillants (conception magico-religieuse). Du fait de la nécessité et de la pénibilité de leur travail, les pré-collecteurs se définissent non comme des miséreux mais comme des gens utiles à la société et jouant un rôle d'une haute importance dans l'assainissement des maisons et de l'environnement de la ville. Les stratégies de revalorisation adoptées par les pré-collecteurs dans le souci de lutter contre la marginalisation consistent en des tournures langagières visant à dépouiller le vocabulaire professionnel des termes désagréables liés aux déchets, en comparaison avec les fonctionnaires moyens sur le plan salarial et en des refus de ramassage. Cette dernière stratégie conduit à des négociations entre les pré-collecteurs et les clients. Ces négociations contribuent à créer et renforcer les liens entre les deux catégories d'acteurs. À travers ces différentes techniques, les pré-collecteurs parviennent à atténuer le stigmate de l'ordure qui ne disparait jamais totalement. Ils se retrouvent donc dans une lutte perpétuelle contre la dévalorisation sociale du travail des déchets, refusant ainsi la relégation aux marges de la société.

\section{References :}

1. Bagalwa, M., Karume, K., Mushagalusa, N. G., Ndegeyi, K., Birali, M., Zirirane, N., ... Bayongwa, C. (2013). Risques potentiels des déchets domestiques sur la santé des populations en milieu rural : cas d'Irhambi Katana (Sud-Kivu, République Démocratique du Congo). Vertigo, 13(2). https://doi.org/10.4000/vertigo.14085 
2. Bercot, R. (2011). De l'art de s'accommoder des épreuves du travail. In D. Corteel \& S. Le Lay (Éd.), Les travailleurs des déchets (p. 207-212). Toulouse: Eres.

3. Biakouyé, K. A. (2014). Lomé au-delà de Lomé : Etalement urbain et territoires dans une métropole d'Afrique sub-saharienne (Thèse de doctorat en géographie). Université de Lomé \& Université Paris Ouest La Défense, Paris.

4. Bouju, J., \& Ouattara, F. (2002). Une anthropologie politique de la fange: conceptions culturelles, pratiques sociales et enjeux institutionnels de la propreté urbaine (Burkina-Faso) (Programme Gestion durable des déchets et de l'assainissement urbain, PS/Eau, PDM). Shadyc-Marseille, Grill-Ouagadougou.

5. Bourguignon, D., \& Herman, G. (2007). Au cœur des groupes de bas statut: La stigmatisation. In G. Herman (Éd.), Travail, chômage et stigmatisation. Une analyse psychosociale (p. 99-44). Louvain-LaNeuve : De Boeck Supérieur.

6. Corteel, D., \& Le Lay, S. (2011). Introduction, Travailler aux abords des déchets : un clair-obscur contemporain. In D. Corteel \& S. Le lay (Éd.), Les travailleurs des déchets (p. 15-32). Toulouse: Eres.

7. Florin, B. (2015). Les récupérateurs de déchets à Casablanca: l'« inclusion perverse» de travailleurs à la marge. Sociologie et sociétés, 47(1), 73-96. https://doi.org/10.7202/1034419ar.

8. Ekoué, A. G. (2019). Le travail des déchets à Lomé : La stigmatisation des précollecteurs d'ordures et des récupérateurs d'objets recyclables. Revue Sociétés \& Économies, (Numéro spécial-Colloque 2018), 2641.

9. Guitard, E. (2012). Le fou, le génie et le tas d'ordures : la passion des déchets comme marqueur de la folie dans la ville de Garoua (Nord Cameroun). In E. Rothmaler, R. Tchokothe, \& H. Tourneux (Éd.), Man and health in the Lake Chad Basin/L'homme et la santé dans le bassin du lac Tchad (p. 91-108). Bayreuth: Rüdiger Köppe Verlag.

10. Guitard, E. (2014). Le grand chef doit être comme le grand tas d'ordures. Gestion des déchets et relations de pouvoir dans les villes de Garoua et Maroua (Cameroun) (Thèse de doctorat en Anthropologie). Université Paris Ouest La Défense, Paris.

11. Guitard, E. (2015). «C'est pas le déchet, c'est le diamant !» Pratiques de récupération et gestion publique des déchets à Garoua et Maroua (Cameroun). In C. Cirelli \& B. Florin (Éd.), Sociétés urbaines et Déchets. Éclairage internationaux (p. 59-86). Tours : Presses Universitaires François Rabelais.

12. Guitard, E. (2016). De « ramasseur d'ordures» à « commandeur de la propreté ». Revaloriser le travail des déchets pour mieux régner 
(Garoua et Maroua, Cameroun). Mouvements des idées et des luttes, Essai et débat. Consulté à l'adresse http://mouvements.info/commandeur-de-la-proprete/

13. Jeanjean, A. (2001). Les égouts en chantier. Présenté à Colloque MàP III (Matières à penser), Université Paris V, Paris, France. Communication inédite <halshs-00004631>.

14. Jeanjean, A. (2011a). Conclusion. Entre transmission, contagion, secret et transgression: ce que l'on se «passe » aux abords des déchets. In D. Corteel \& S. Le Lay (Éd.), Les travailleurs des déchets (p. 279-302). Eres.

15. Jeanjean, A. (2011b). Travailler à la morgue ou dans les égouts. Ethnologie française, XLI(1), 59-66.

16. Koledzi, K. E. (2011). Valorisation des déchets solides urbains dans les quartiers de Lomé (Togo): Approche méthodologique pour une production durable de compost (Thèse de doctorat en chimie et microbiologie de l'eau). Université de Lomé \& Université de Limoges, Limoges.

17. Lhuillier, D. (2005). Le sale boulot. Travailler, 2(14), 73-98.

18. Lhuillier, D. (2011). Souillure et transgression : le travail sur le négatif psychosocial. In D. Corteel \& S. Le Lay (Éd.), Les travailleurs des déchets (p. 33-43). Toulouse: Eres.

19. Morlet, T. (2011). La pénibilité au travail des équipiers de collecte dans le secteur privé : éléments organisationnels et managériaux. In D. Corteel \& S. Le Lay (Éd.), Les travailleurs des déchets (p. 153-167). Toulouse: Eres.

20. Lelo Nzuzi, F. (2008). Kinshasa, ville et environnement. Paris: L'Harmattan.

21. Poulsen, O. M., Breuma, N. 0., Ebbehoj, N., Hansen, A. M., Ivens, U. I., van Lelieveld, D., ... Wilkins, K. C. (1995). Collection of domestic waste. Review of occupational health problems and their possible causes. The Science of the Total Environment, (170), 1-19. https://doi.org/10.1016/0048-9697(95)04524-5

22. Philippot, E. (1997). Gestion et pratiques des déchets solides à Pondichéry. Dynamiques et dégradations des espaces en milieu urbain. In D. Bley, J. Champaud, P. Baudot, B. Brun, H. Pagezy, \& N. Vernazza-Licht (Éd.), Villes du Sud et environnement (p. 100-109). Éditions de Bergier-Travaux de la Société d'Écologie Humaine.

23. Poirée, J. (2004). Observer le pouvoir. Le Portique, (13-14). Consulté à l'adresse http://leportique.revues.org/630

24. Soares, A. (2011). L'élégance des éboueurs. In D. Corteel \& S. Le Lay (Éd.), Les travailleurs des déchets (p. 213-234). Toulouse: Eres. 
25. Tajfel, H., \& Turner, J. (1979). An integrative theory of intergroup conflict. In S. Worchel \& W. Austin (Éd.), The social psychology of intergroup relations (p. 33-47). Pacific Grove, CA : Brooks/Cole.

26. Tiankpa, Y. (2016). Insalubrité et gestion des déchets ménagers au Togo (Thèse de doctorat en géographie humaine). Université de Lomé, Lomé.

27. Zonabend, F. (1999). L'innommable et l'innomé. In J.-C. Beaune (Éd.), Le déchet, le rebut, le rien (p. 90-98). Seyssel: Champ Vallon. 